\title{
Coulomb dissociation
}

\section{another "Trojan Horse"}

\author{
Tohru Motobayashi ${ }^{1, \star}$
}

${ }^{1}$ RIKEN Nishina Center, 2-1 Hirosawa, Wako, Saitama 351-0198, Japan

\begin{abstract}
Coulomb dissociation is discussed in connection with the Trojan-Horse method and other quasi-free reactions.
\end{abstract}

\section{$1{ }^{1} \mathrm{H}\left(\mathrm{d},{ }^{2} \mathrm{He}\right) \mathrm{n}$ reactions - a Quasi Free Reaction Process}

The Trojan-Horse method relies on the "quasi-free reaction" mechanism involved in a direct reaction with three particles in the final state. Claudio Spitaleri and his group in Catania have developed this method to a level at which reliable astrophysical reaction rates are extracted. They often adopt a simplest approximation for the quasi-free process, PWIA (Plane Wave Impulse Approximation), with successful results [1].

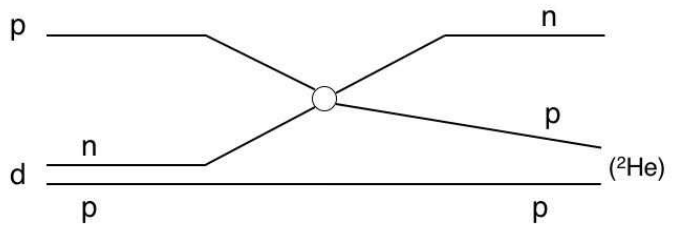

Figure 1. Diagram for the quasi-free process for the ${ }^{1} \mathrm{H}\left(\mathrm{d},{ }^{2} \mathrm{He}\right) \mathrm{n}$ reaction. The three-body final state is dominated as a final-state interaction by the strong ${ }^{1} \mathrm{~S}_{0}$ enhancement in the proton-proton interaction.

The ${ }^{1} \mathrm{H}\left(\mathrm{d},{ }^{2} \mathrm{He}\right) \mathrm{n}$ reaction at an intermediate energy may also be dominated by the quasi-free reaction process, where the two-body process in question is the charge exchange scattering by the neutron in the projectile deutron and the target proton (see Fig. 1). The symbol ${ }^{2} \mathrm{He}$ here denotes a system of two protons in their unbound singlet $\mathrm{S}$ state, with a strong enhancement at around $400 \mathrm{keV}$ relative energy. The p-n charge-exchange could be viewed as a process exchanging a (virtual) pion, and thus the entire process is analogous to the Trojan-Horse process with the pion as the "soldier". The data taken at Saturne in late 1980s using $200 \mathrm{MeV}$ polarized deuteron beams were analyzed with PWIA [2]. The tensor analyzing powers and even the absolute magnitudes of the differential cross sections have been successfully explained by assuming a $n+p \rightarrow p+n$ quasi-free process at small momentum transfers. In PWIA calculations, charge exchange nucleon-nucleon amplitudes are employed to predict

^e-mail: motobaya@riken.jp 
spin-dependent observables [3]. That was our surprise, because nucleon rearrangement reactions in this energy regime are not always well described by such a simple treatment. The major purpose of this study was to establish the reaction mechanism, with its practical application to the measurements of the deuteron tensor polarization. It should be noted that the quasi-free assumption works well only at small momentum-transfers, in which charge exchange or pion-exchange dominates the $n+p$ interaction. That is similar to the Trojan-Horse applications, where the kinematical conditions are selected to assure the quasi free mechanism.

\section{Coulomb Dissociation}

Many radiative proton-capture reactions play key roles in astrophysical hydrogen burning [4]. Their direct measurements are often affected by the small cross sections. Difficulty of preparing radioactive targets could be the serious concern for the cases involving unstable nuclei. The Coulomb dissociation provides an indirect method for determination of radiative capture reactions [5, 6]. In a Coulomb dissociation experiment, the residual nucleus $\mathrm{A}$ of the reaction $\mathrm{B}(\mathrm{x}, \gamma) \mathrm{A}$ bombards a high- $Z$ target as lead, and it is excited in the Coulomb field to its unbound state that decays to the $\mathrm{B}+\mathrm{x}$ channel. The Coulomb dissociation process is regarded as absorption of a virtual photon, $\mathrm{A}(\gamma, \mathrm{x}) \mathrm{B}$, the inverse of the radiative capture of interest, and therefore the dissociation yield can be related to the relevant capture cross section.

Figure 2 schematically shows the Coulomb dissociation process. When a projectile nucleus $\mathrm{A}$ is passing the vicinity of a high $Z$ nucleus, the projectile feels the presence of the electric field generated by the high $Z$ nucleus. The time-dependent amplitude of the electric field (shown in the bottomleft of Fig. 2) can be decomposed in electromagnetic waves with different frequencies, and hence is equivalent to the exposition of nucleus A to photons with different energies. The photon spectrum is obtained by the Fourier transform of the impulse. With fast beams of $\approx 50 \mathrm{MeV} /$ nucleon or higher, the maximum energy of the virtual photons can cover the range of excitation energy of the nucleus A in most of the cases of astrophysical interest [5].

Application of the Coulomb dissociation process to astrophysical radiative capture has been discussed by Baur, Bertulani and Rebel [5] for the first time, and some review articles are available [6, 7]. The photo-absorption is an inverse process of the radiative capture, their cross sections are related by the detailed balance as,

$$
\sigma_{\mathrm{A}+\gamma \rightarrow \mathrm{B}+x}=\frac{\left(2 j_{\mathrm{B}}+1\right)\left(2 j_{\mathrm{x}}+1\right)}{2\left(2 j_{\mathrm{A}}+1\right)} \frac{k^{2}}{k_{\gamma}^{2}} \sigma_{\mathrm{B}+x \rightarrow \mathrm{A}+\gamma} .
$$

The spins of A, B and $\mathrm{x}$ are denoted by $j$ 's, respectively. The symbols $k$ and $k_{\gamma}$ denote the wave numbers in the $\mathrm{B}+\mathrm{x}$ channel and $\mathrm{A}+\gamma$ channel, respectively. Due to the large phase space volume for the $\mathrm{B}+\mathrm{x}$ channel, the photo-absorption cross section is enhanced. For example the wave-number ratio $k^{2} / k_{\gamma}^{2}$ in Eq. 1 is calculated to be about 1000 for the case of ${ }^{7} \mathrm{Be}(\mathrm{p}, \gamma)^{8} \mathrm{~B}$ reaction. This enables experimental studies on explosive burning with beams of unstable nuclei or radioactive isotope (RI) beams, the intensity of which is expected to be low.

The first experimental result with RI beams was obtained at RIKEN on the ${ }^{13} \mathrm{~N}(\mathrm{p}, \gamma){ }^{14} \mathrm{O}$ reaction, relevant to the hot CNO cycle nuclear burning [9]. This work was published in 1991, almost at the same time and on the same subject as the experiment at Leuvain la Neuve [10] using a radioactive ${ }^{13} \mathrm{~N}$ beam for a direct measurement. The Coulomb dissociation experiment used fast RI beams of ${ }^{14} \mathrm{O}$ at 87.5 MeV/nucleon. The results of these two experiments agreed with each other. The higher statistics of the Coulomb dissociation experiment demonstrate the above mentioned advantage of the method.

The solar-neutrino production process ${ }^{7} \mathrm{Be}(\mathrm{p}, \gamma)^{8} \mathrm{~B}$ has been studied by the Coulmob dissociation method in several laboratories [11-13]. In the first experiment at RIKEN, radioactive ${ }^{8} \mathrm{~B}$ nuclei were 

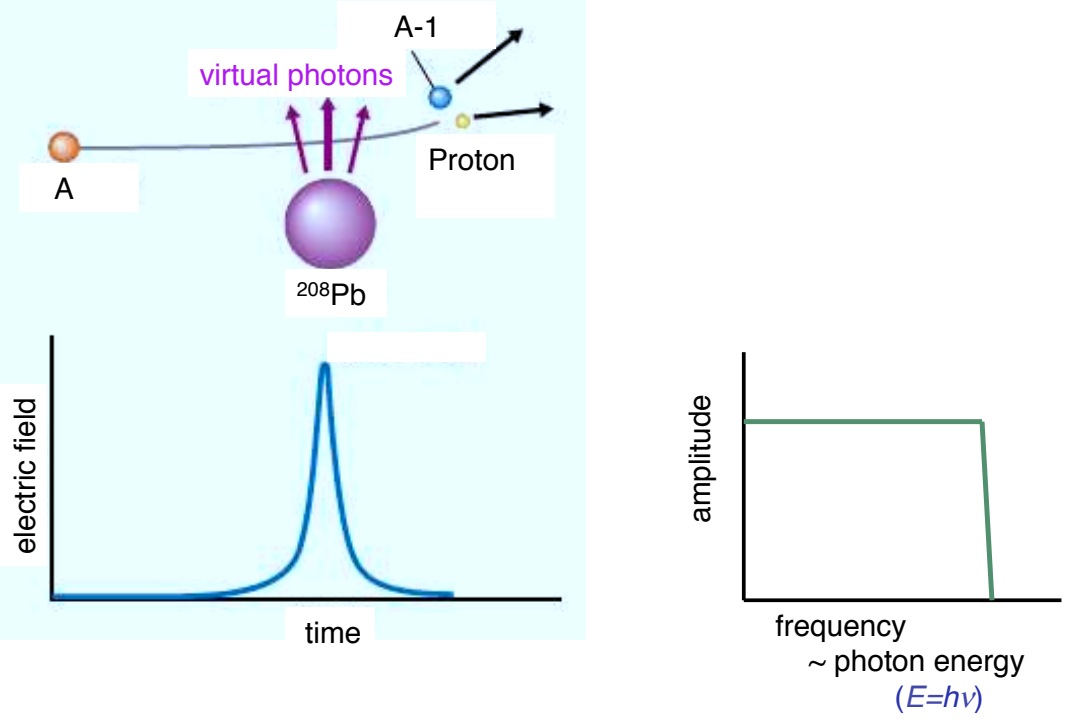

Figure 2. Schematic picture of the Coulomb dissociation. The left and right lower panels show the electric force felt by the projectile as a function of time and its Fourier transform, respectively. This figure is originally from ref. [8]

produced by the ${ }^{12} \mathrm{C}+{ }^{9} \mathrm{Be}$ fragmentation reaction with a $92 \mathrm{MeV} /$ nucleon ${ }^{12} \mathrm{C}$ primary beam, then analyzed and transported to a ${ }^{208} \mathrm{~Pb}$ secondary target. The energies and directions of the fragments were measured to obtain the $\mathrm{p}^{-7} \mathrm{Be}$ relative energy event-by-event, with a $\Delta E-E$ plastic scintillator hodoscope $[7,11]$.

Figure 3 shows the astrophysical $S_{17}$-factors obtained in the experiments at RIKEN together with the results from several direct $(\mathrm{p}, \gamma)$ measurements. Our Coulomb dissociation data agree well with the results by Filippone et al. [19] and Vaughn et al. [17]. Direct data taken in 2000s by Junghans et al. [22] and Baby et al. [23] (not shown in the figure), are slightly larger than the ones shown by the theoretical curves, which are normalized to the second Colomb dissociation data. The comparison between the results by the direct capture and Coulomb dissociation provided useful information on this important reaction, since the two methods are independent, and their sources of possible systematic uncertainties are different [7].

The Coulomb dissociation is also a process with a three body final state. As indicated in Fig. 2, the virtual photon induces the breakup of the nucleus A. Therefore the Coulomb dissociation may be another analogue of Trojan-Horse reaction with the "soldier" of the virtual photon. In contrast to the Trojan-Horse applications, the process is theoretically treated often as inelastic excitation of the nucleus A to its excited state above the particle decay threshold. Another difference lies in the 


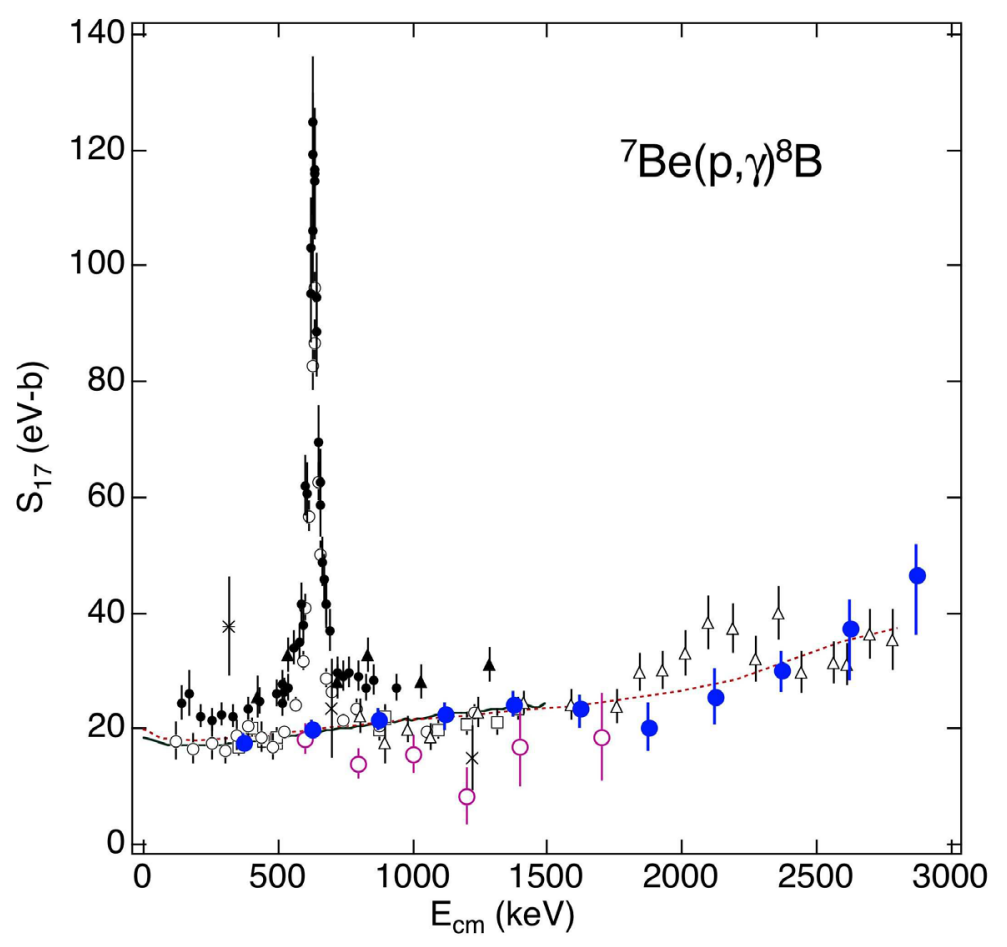

Figure 3. Astrophysical $S_{17}$-factors for the ${ }^{7} \mathrm{Be}(\mathrm{p}, \gamma)^{8} \mathrm{~B}$ reaction extracted from the first (large open circles in purple) [11] and second (large solid circles in blue) [12] experiments at RIKEN. Direct (p, $\gamma$ ) data by Kavanagh [14] (crosses), Parker [15] (closed triangles) Kavanagh et al. [16] (closed circles), Vaughn et al. [17] (open triangles), Wiezorek et al. [18] (asterisk), and Filippone et al. [19] (open circles) are also shown. The energy dependent $S_{17}$ factors theoretically predicted by Barker and Spear [20] (solid curve) and Descouvemont and Baye [21] are shown. They are normalized to the data from the second Coulomb dissociation experiment.

incident energy. The Coulomb dissociation method is applied at relatively high energy to make the virtual photon capable to excite the nucleus to its unbound state. Both the methods have a common feature of yield enhancement. The Trojan-Horse method takes this advantage to mostly overcome the low cross section of the relevant reaction of astrophysical interest, while the Coulomb dissociation is often applied to a capture reaction involving a short-lived nucleus, the beam intensity of which is very low.

\section{Summary and Remarks}

Two examples of the reactions having three particles in the final states are considered. As well as the success for various Trojan Horse reactions thoroughly studied by Claudio Spitaleri and his Catania group, PWIA well describes the ${ }^{1} \mathrm{H}\left(\mathrm{d},{ }^{2} \mathrm{He}\right)$ n reaction data including the differential cross sections and tensor analyzing powers. The Coulomb dissociation is another indirect method for studying astrophysical reactions. Its mechanism can be regarded as absorption of a virtual photon, which plays a similar role of the "soldier" in a Trojan Horse reaction. Thus the inverse process, radiative capture, can be studied by the Coulomb dissociation. Results of Coulomb dissociation studies agree with 
relevant direct data, demonstrating its usefulness. It should be noted that the Coulomb dissociation can indirectly study radiative capture, often of importance in nucleosynthesis, while the Trojan Horse method can indirectly access particle transfer reactions of astrophysical interest.

These successful applications of simple theoretical treatments to various types of reactions with three-body final state lead me a "feeling": the quasi-free mechanism or first-order Coulomb excitation can naturally find a way to particle-unbound final state, providing a simple and reliable tool for analysis. On the other hand, population of discrete bound-states requires more kinematically restricted conditions and may allow for complicated mechanism to be involved. That is only a prejudice, but we may thank this favorable situation.

The author of this article conducted several Coulomb dissociation experiments in the period when Spitaleri and his group were vigorously studying and establishing the Torojan Horse method. We appreciate their long experimental efforts and careful analysis to extract the relevant cross sections of astrophysical interest.

\section{References}

[1] C. Spitaleri et al., Phys. Rev. C 60, 055802 (1999); S. Typel and G. Baur, Annals Phys. 305, 228 (2003); C. Spitaleri et al., Eur. Phys. J. A 52, 77 (2016)

[2] T. Motobayashi et. al., Phys. Lett. B 233, 69 (1989); S. Kox et. al., Nucl. Phys. A 556, 621 (1993)

[3] D.V. Bugg and C. Wilkin, Phys. Lett. B 152, 37 (1985)

[4] E.M. Burbidge, G.R. Burbidge, W.A. Fowler, and F. Hoyle, Rev. Mod. Phys. 29, 547 (1957); C. Rolfs and W. Rodny, Cauldrons in the Cosmos (University of Chicago Press, 1988)

[5] G. Baur, C.A. Bertulani and H. Rebel, Nucl. Phys. A 458, 188 (1986).

[6] G. Baur and H. Rebel, J. Phys. G 20, 1 (1994); Ann. Rev. Nucl. and Part. Sci. 46, 321 (1996).

[7] T. Motobayashi, Nucl. Phys., A 693, 258 (2001).

[8] T. Motobayashi and H. Sakurai, Prog. Theor. Exp. Phys. 2012, 03 C001 (2012).

[9] T. Motobayashi et al., Phys. Lett. B 264, 259 (1991).

[10] P. Decrock et al., Phys. Rev. Lett. 67, 808 (1991).

[11] T. Motobayashi et al., Phys. Rev. Lett., 73, 2680 (1994)

[12] T. Kikuchi et al., Phys. Lett. B 391, 261 (1997)

[13] N. Iwasa et al., Phys. Rev. Lett. 83, 2910 (1999); B. Davis et al., Phys. Rev. Lett. 86, 2750 (2001) F. Schümann et al., Phys. Rev. Lett. 90, 232501 (2003);

[14] R.W. Kavanagh, Nucl. Phys. 15, 411 (1960)

[15] P.D. Parker, Phys. Rev. 150, 851 (1966)

[16] R.W. Kavanagh, T.A. Tombrello, J.M. Mosher and D.R. Goosman, Bull. Am. Phys. Soc. 14, 1209 (1969)

[17] F.J. Vaughn, R.A. Chalmers, D. Kohler, and L.F. Chase, Jr., Phys. Rev. C 21657 (1970).

[18] C. Wiezorek, H. Kräwinkel, R. Santo, and L. Wallek, Z. Phys. A 282, 121 (1977)

[19] B. Filippone, S.J. Elwyn, C.N. Davids, and D.D. Koetke, Phys. Rev. Lett., 50, 412 (1983); Phys. Rev. C 28, 2222 (1983).

[20] F.C. Barker and R.H. Spear, Astrophys. J. 307, 847 (1986)

[21] P. Descouvemong and D. Baye, Nucl. Phys. A 567, 341 (1994)

[22] A.R. Junghans et al., Phys. Rev. Lett. 86, 041101 (2002) .

[23] L.T. Baby et al., Phys. Rev. Lett. 90, (2003) 022501; Phys. Rev. C 67065805 (2003). 
